\title{
Effects of Hydrophobic Solvents on X-Ray Diffraction Patterns of Regenerated Cellulose Membrane
}

\author{
Kazuishi $\mathrm{SATO}^{\dagger}$, Hisaya MochizUKI, Kunihiko OKAJIMA ${ }^{\dagger}$, and Chihiro YAmane, ${ }^{* \dagger}$ \\ Central Research Laboratories, Asahi-Kasei Corporation, 1-2 Samejima, Fuji 416-8501, Japan \\ *Department of Domestic Science, Kobe Women's University, 2-1 Higashisuma-Aoyama, Suma-ku, Kobe 654-8585, Japan
}

(Received November 28, 2003; Accepted February 10, 2004; Published June 15, 2004)

\begin{abstract}
Specific interactions between cellulose and hydrophobic solvents were studied using highly planar oriented cellophane and X-Ray diffraction analysis. The planar orientation index $\left(f_{\|(1 \overline{1} 0)}\right)$ was found to decrease considerably by hydrophobic solvent treatment. Toluene, a hydrophobic solvent, caused decrease at specific concentration in $f_{\|(1 \overline{1} 0)}$ at toluene content of $c a$. 20-40 wt \% to cellulose with keeping its crystallinity and the degree of intermolecular hydrogen bonding. The molecular sheet-like assembly (corresponding to $\alpha_{\mathrm{sh}}$ relaxation), proposed previously by Yamane et al. [Polym. J., 28, 1039 (1996)] may be a plausible explanation of the result. Analysis of X-Ray diffraction intensity of cellulose/solvent systems might give some information on the extent of interaction between cellulose and solvent in question. However, whether such change in specific solvent concentration with orientation index is related to the long-periodicity change, as observed for water, should be studied. [DOI 10.1295/polymj.36.478]

KEY WORDS Cellulose / Planar Orientation / Hydrophobic Solvent / X-Ray Diffraction / Crystal Plane / Molecular Sheet /
\end{abstract}

Water causes a sudden long-periodicity change of regenerated cellulose fiber at $c a .30 \mathrm{wt} \%{ }^{1}$ Such concentration-specific structural change for natural cellulose was also observed indirectly by $\mathrm{T}_{1}$ measurement of water in cellulose. ${ }^{2}$ Ago et al. also showed that amorphous cellulose is reorganized to cellulose II crystals by ball-milling preferentially when water content is $c a$. 30\%. ${ }^{3}$ Hydrophobic solvents such as hexane, toluene and dichloromethane are captured in cellulose domain even after some severe vacuum drying. ${ }^{4}$ Such nonpolar solvents have been frequently utilized as heterogeneous reaction media for converting cellulose to its derivatives. Marchenko et al. ${ }^{5}$ studied structural changes in cellulose (wood pulp, cotton) caused by dichloromethane (DCM) by using X-Ray diffraction, IR, and SEM analyses, and noted intrafibrillar swelling, no practical change in X-Ray crystallinity, some changes in $\mathrm{CH}_{2}$ vibration (IR; 1380 $\mathrm{cm}^{-1}$ ) absorption, a loosening of cellulose structure by penetration of DCM into nonordered region and formation of rigid cellulose structure after removal of DCM. Such studies are quite important, although not much research seems to have been done. Recently, we have reported the mechanical molecular alloy preparation of cellulose/thermoplastics in the presence of such a nonpolar solvent. ${ }^{6,7}$ The method is based on the facts that 1) such solvents lowers the temperature (a kind of $T_{\mathrm{g}}$ ) considerably, representing $\alpha_{\text {sh }}$ relaxation mode (inner phase relaxation of cellu- lose main chains on molecular sheet-like assembly 8,9 on $\tan \delta-T$ curves, from $c a .170^{\circ} \mathrm{C}$ to less than room temperature ${ }^{9}$ and 2) such solvents might induce concentration-specific mobilization of cellulose chains. Therefore, more detailed studies on the interaction of cellulose with nonpolar solvents are immediately required. Recently, a concentration-specific interaction between cellulose with hydrophobic solvent (toluene) was noted by Ago et al., ${ }^{10}$ by measuring the spin-lattice relaxation $T_{1}$ of specific proton NMR peak of cellulose. Concentration-specific interaction took place between toluene content of $c a$. 20-30 wt $\%$ for cellulose, and dioctyl phthalate (DOP) specifically changed the mobility of cellulose at 20$40 \mathrm{wt} \%$ for cellulose. ${ }^{11}$ These studies concentrate only on natural fibrous or powdery samples, and whether such hydrophobic interaction takes place for regenerated cellulose is of our interest.

\section{EXPELIMENTAL}

\section{Samples}

A commercial semipermeable cellophane (thickness; $0.0508 \mathrm{~mm}$, average pore size; $50 \AA$, $\alpha$-cellulose content; $99 \%$, manufactured by Viskase Companies, Inc.). The cellophane was first dipped in ionexchanged water, and then dried under vacuum at $60{ }^{\circ} \mathrm{C}$ for $6 \mathrm{~h}$. A given amount of toluene $(0-60 \mathrm{wt} \%$ for cellulose) was sprayed on the dried cellophane

\footnotetext{
${ }^{\dagger}$ Present Address: Faculty of Engineering, Tokushima Bunri University, 1314 Shido, Sanuki 769-2193, Japan.

${ }^{\dagger \dagger}$ To whom correspondence should be addressed (E-mail: yamane@suma.kobe-wu.ac.jp).
} 
and the each treated cellophane was stocked in a sealed vessel at $25^{\circ} \mathrm{C}$ for $2 \mathrm{~h}$ and $5 \mathrm{~d}$ and then subjected to X-Ray diffraction measurement. For irradiation of X-Ray to the parallel direction of cellophane surface, several pretreated cellophones are piled and roughly pressed. In the case of cyclohexane, hexane, carbon tetrachloride, acetone, ethyl acetate and bis2-ethyl-hexyl phthalate (DOP), similar sample preparation $(2 \mathrm{~h}$ treatment) was made for the cellophanes with $40 \mathrm{wt} \%$ of each solvent. All Solvents used were guaranteed grade.

\section{Measurement}

X-Ray diffraction patterns of samples were measured by reflection method and recorded on a X-Ray diffractometer with scintillation counter (RigakuRU200B; $\mathrm{Cu}-\mathrm{K} \alpha)$. X-Ray was irradiated at $\theta^{\circ}(2 \theta=$ $5-35^{\circ}$ ) to the membrane surface (parallel incidence) and its cross section (perpendicular incidence). Measurement was made at $1^{\circ} / \mathrm{min}$ with inner reference $\left(\mathrm{SiO}_{2} ; 2 \theta=28.45^{\circ}\right)$. To calculate the orientation of crystal planes (110), diffraction intensity of parallel incidence $\left(I_{(1 \overline{1} 0)}\right)$ at $2 \theta=c a \cdot 12^{\circ},\left(I_{(110)}\right)$ at $2 \theta=c a$. $20^{\circ}$ and $\left(I_{(020)}\right)$ at $2 \theta=c a .22^{\circ}$ was measured. XRay diffraction pattern of random oriented sample was recorded by the powder method. Diffraction intensity of the random oriented sample (i) of each crystal plane was measured. It is well known that the (110) crystal plane is orientated parallel to the film surface in case of cellophane. Orientation index, $f_{\|(1 \overline{1} 0)}$ for (110) plane, was evaluated using the simple method reported by Takahashi ${ }^{12}$ as follows. Orientation index shows relative degree of planar orientation and takes 0 (random) as 1 (parallel to surface).

$$
\begin{aligned}
f_{\|(1 \overline{1} 0)}= & \left\{I_{(1 \overline{1} 0)}-\left(I_{(110)+(020)}\right.\right. \\
& \left.\left.\times i_{(1 \overline{1} 0)} / i_{(110)+(020)}\right)\right\} / I_{(1 \overline{1} 0)}
\end{aligned}
$$

Apparent crystal size (ACS) was estimated through use of Scherrer's equation. ${ }^{13}$

$$
\mathrm{ACS}=0.9 \lambda / \cos \theta \cdot \beta
$$

with

$$
\beta=\left(B^{2}-b^{2}\right)^{1 / 2}
$$

where $\lambda$ is the wavelength of the incident X-Ray $(1.5418 \AA), \theta$ is the diffraction angle corresponding to the (110) plane, $b$ is the instrumental constant $(0.2)$ and $B$ is the half-value width, in radians, of the peak angle of the (110) plane.

Here, the unit cell parameters of cellulose II, $a=$ $8.10 \AA, b=9.03 \AA, c=10.31 \AA$, and $\gamma=117.10^{\circ}$ were used. ${ }^{14}$

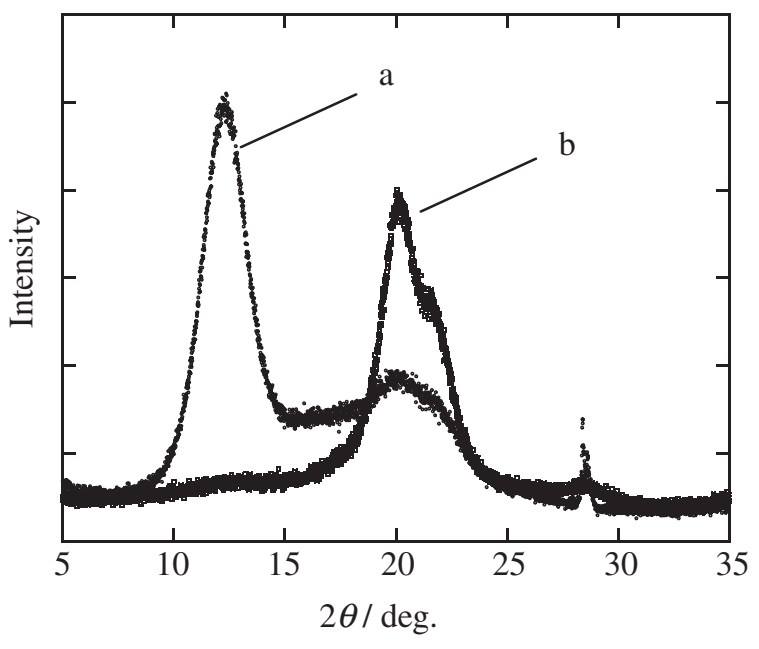

Figure 1. X-Ray diffraction patterns of dried original cellophane: a, obtained by parallel incidence to the cellophane surface; $\mathrm{b}$, perpendicular incidence to the cellophane surface.

\section{RESULTS AND DISCUSSION}

Figure 1 shows X-Ray diffraction patterns of original dried cellophane. X-Ray diffraction obtained by perpendicular incidence to the cellophane surface shows 1) strong (110) plane peak at $2 \theta=c a \cdot 20^{\circ}$, 2) second conspicuous peak ((020) plane) at $2 \theta=$ ca. $22^{\circ}$, and 3) practically no (110) plane peak at $2 \theta=c a .12^{\circ}$. In contrast, X-Ray diffraction pattern obtained by parallel incidence to the cellophane surface showed a strong (110) plane peak at $2 \theta=$ ca. $12^{\circ}$. Theoretical diffraction strength for (110) crystal plane is $c a .1 / 3$ of those for (110) and (020). The (110) crystal plane is thus orientated parallel to the film surface. Density of hydroxyl group on the surface of (110) plane is quite high. ${ }^{15}$ Such cellulose membrane generally shows very low contact angle of water droplet, high wettability with high anisotropic swelling, leading to macroscopic structural deformation on mechanical impact, such as easy fibrillation in wet state and lowering of crystallinity in dry state.

Table I summarizes diffraction peak positions for three planes, relative intensity of (110) and (020) plane compared to (110) peak intensity for toluenetreated $(2 \mathrm{~h})$ sample, and planar orientation index $f_{\|(1 \overline{1} 0)}$ for $2 \mathrm{~h}-$ and $5 \mathrm{~d}$-treated samples. Note that ACS of the (110) plane for all data points is equivalent in value.

Figure 2 shows typical X-Ray diffraction patterns obtained by parallel incidence to the cellophane surface of the toluene-treated $(2 \mathrm{~h})$ cellophanes. Table I shows that the (110) plane peak at $2 \theta=c a .12^{\circ}$ decreased by toluene intrusion compared to other planes. Inspection of the correlation between RI for (110) and RI for (020) showed that relative intensity was main- 
Table I. X-Ray parameters for cellophane treated with toluene

\begin{tabular}{|c|c|c|c|c|c|c|c|c|c|}
\hline \multirow{2}{*}{$\begin{array}{l}\text { Toluene } \\
\text { content }\end{array}$} & \multicolumn{2}{|c|}{$(1 \overline{1} 0)$} & \multirow[b]{2}{*}{$\mathrm{RI}^{\mathrm{a}}$} & \multicolumn{2}{|c|}{ (110) } & \multicolumn{2}{|c|}{$(020)$} & \multirow{2}{*}{$\begin{array}{c}f_{\|(1 \overline{1} 0)} \\
\text { after } 2 \mathrm{~h}\end{array}$} & \multirow{2}{*}{$\begin{array}{c}f_{\|(1 \overline{1} 0)} \\
\text { after } 5 \mathrm{~d}\end{array}$} \\
\hline & $2 \theta(\mathrm{deg})$ & $\operatorname{ACS}(\AA)$ & & $2 \theta(\mathrm{deg})$ & $\mathrm{RI}^{\mathrm{a}}$ & $2 \theta(\mathrm{deg})$ & $\mathrm{RI}^{\mathrm{a}}$ & & \\
\hline 0 & 12.32 & 36.5 & 100 & 20.48 & 20.5 & 21.74 & 17.8 & 0.956 & 0.956 \\
\hline 10 & 12.06 & 34.9 & 100 & 20.71 & 26.7 & 22.00 & 30.0 & 0.934 & 0.926 \\
\hline 20 & 12.06 & 34.9 & 100 & 20.59 & 37.5 & 21.75 & 38.6 & 0.911 & 0.907 \\
\hline 40 & 12.17 & 34.9 & 100 & 20.50 & 57.9 & 21.87 & 59.6 & 0.866 & 0.931 \\
\hline 60 & 12.27 & 34.9 & 100 & 20.44 & 31.7 & 21.79 & 31.7 & 0.931 & 0.926 \\
\hline
\end{tabular}

${ }^{\mathrm{a}} \mathrm{RI}$ is relative intensity compared to (110) intensity.

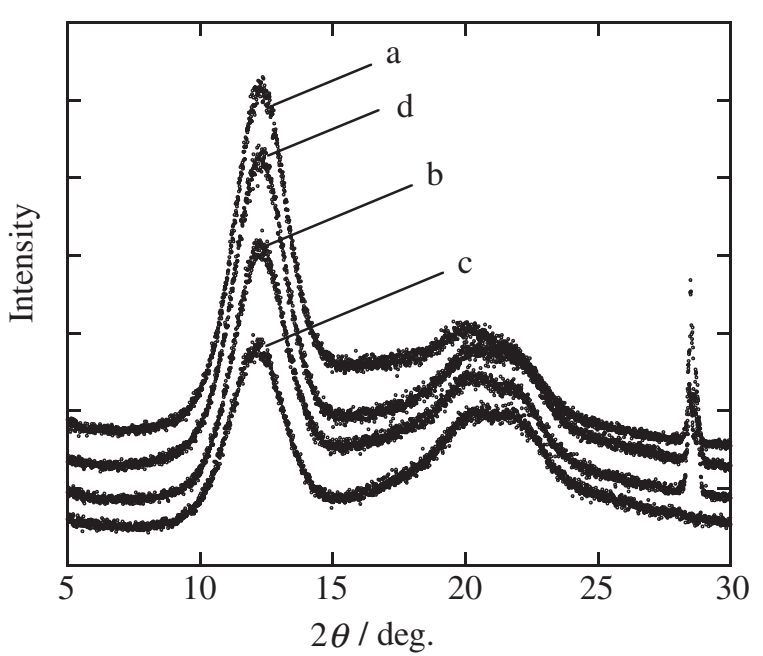

Figure 2. X-Ray diffraction patterns obtained by parallel incidence to the cellophane surface of the toluene-treated $(2 \mathrm{~h})$ cellophanes: a, dried original cellophane; $\mathrm{b}$, toluene content was $20 \mathrm{wt} \%$; c, 40 wt \%; d, $60 \mathrm{wt} \%$.

tained almost constant, indicating no loss in crystallinity. This is supported by the fact that crystallinity of the powdered natural cellulose does not change significantly even by ball-milling with toluene, as pointed out previously. ${ }^{8,10}$ Thus, the lowering of (110) intensity should be correlated with lowering crystal planar orientation. However, the lowering of $f_{\|(1 \overline{1} 0)}$ also should be correlated with increasing of (110) and (020) plane intensity, from a crystalline view point.

Such lowering of specific plane may be explained by relaxation of the (110) plane. Since the (110) plane is formed by hydrophobic interaction, ${ }^{9}$ toluene may strongly affect the crystal plane, and relaxing the hydrophobic interaction of the plane, and finally, causing some distribution in (110) spacing which should have $4.4 \AA$ or $4.1 \AA$ periodicity. This explanation is supported by the fact that molecular sheet-like assembly is also relaxed by toluene treatment. ${ }^{9}$ The sheet-like assembly is assumed to be one of the amorphous components $^{16}$ (corresponding to $\alpha_{\mathrm{sh}}$ relaxation) and derive from (110) crystal plane. ${ }^{9}$ As the result, intensities of (110) and (020) planes with spacings of $4.4 \AA$ and $4.1 \AA$ respectively might be reduced irrespective of the orientation index $f_{\|(1 \overline{1} 0)}$ and crystallinity. The

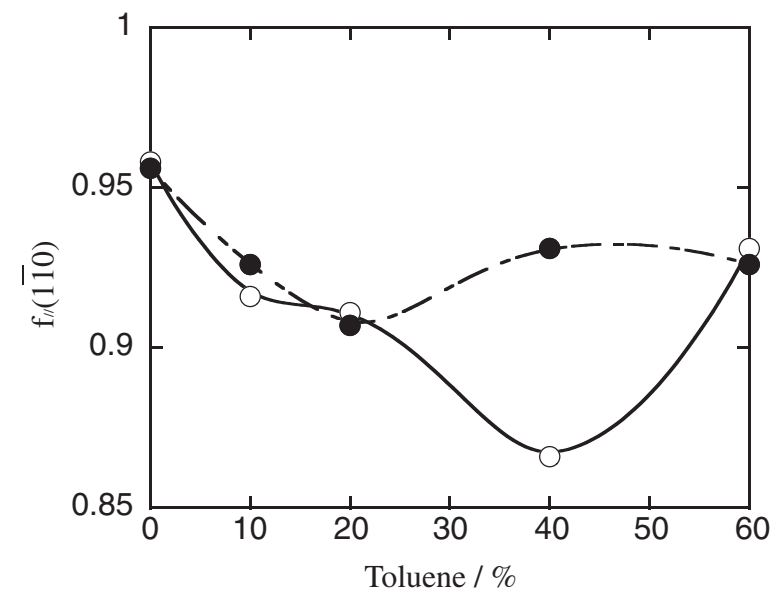

Figure 3. Effects of toluene content on the orientation index $f_{\|(1 \overline{1} 0)}: \bigcirc, 2$ h-toluene treatment; $\bullet, 5$ d-toluene treatment.

lowering of (110) intensity was more remarkable than (020). This may be related to the surface energy of each crystal plane. (110) plane may easily be affected in toluene, since the surface energy of the plane is lower than (020) plane. ${ }^{17}$

Figure 3 shows plots of $f_{\|(1 \overline{1} 0)} v s$. toluene content for the treated cellophane $(O ; 2 \mathrm{~h}$ treatment, $\bullet ; 5 \mathrm{~d}$ treatment). Clearly, ca. $40 \%$ toluene is specific for the $2 \mathrm{~h}$-treated sample and $c a .20 \mathrm{wt} \%$ toluene is specific for $5 \mathrm{~d}$-treated sample. At specific points, $f_{\|(1 \overline{1} 0)}$ becomes minimum. The reason for the shift of specific concentration to lower concentration by prolonged of treatment time may correlate with penetration time of effective enough toluene to cause such decrease in plane orientation. $f_{\|(1 \overline{1} 0)}$ of $2 \mathrm{~h}$-treated sample was much smaller than $5 \mathrm{~d}$-treated sample at $40 \%$ toluene. Toluene lowers glass transition temperature to less than room temperature ${ }^{18}$ and activates molecular motion in the amorphous region. Some structural formation may be generated under these conditions. Such observation might not be possible for powdered cellulose samples. It is not clear at present whether similar long-periodicity change occurs as observed for water (specific concentration; $c a .30 \mathrm{wt} \%$ ).

Although exact diffraction angles could not be determined, those for (110) and (110) planes seem to 


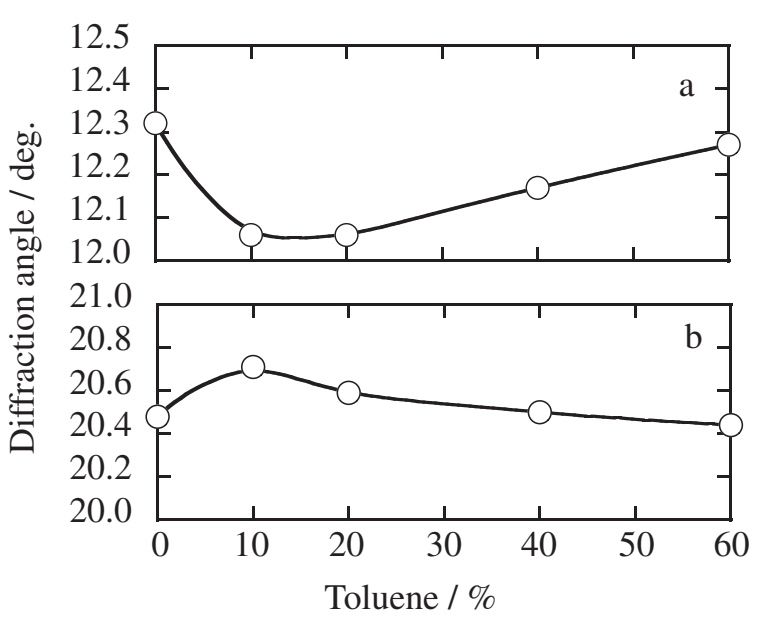

Figure 4. Effects of toluene content on diffraction angles of (110) and (110) planes: a, (11̄0) plane; b, (110) plane.

shift reversely as a function of toluene content (TC), as shown in Figure $4 a$ and $b$. That is to say, the angle for the (110) plane slightly shifts to a lower one, approaching minimum at toluene content $=10-20$ wt $\%$, then shifts to a higher one, and also the angle for (110) slightly shifts to large one, approaching maximum at toluene content $=c a .20 \mathrm{wt} \%$, and finally shifts to higher angle. Thus, (110) and (110) planes are slightly widen and narrowed, respectively, at specific concentration of toluene. However, $f_{\|(1 \overline{1} 0)}$ seems not to be correlated with refraction angles representing (110) and (110) planes. ACS proved it does not change at all. Crystallinity and the degree of intermolecular hydrogen bonds may thus be slightly changed during toluene treatment. Deviations of unit cell parameters, $a, b$, and $\gamma$ from original cellulose sample remained within $1.5 \%$ or less after toluene treatment.

Figure 5 shows X-Ray diffraction patterns for cellophane treated with several hydrophobic solvents. Although the solvent concentration giving specific structural change of cellulose, if any, might be different from solvent to solvent, the diffraction patterns are given at constant concentration here $(40 \mathrm{wt} \%$ to cellophane). However, as seen in the figure, diffraction intensities are all different depending on the kind of solvent used. This means that solvent is vaporized

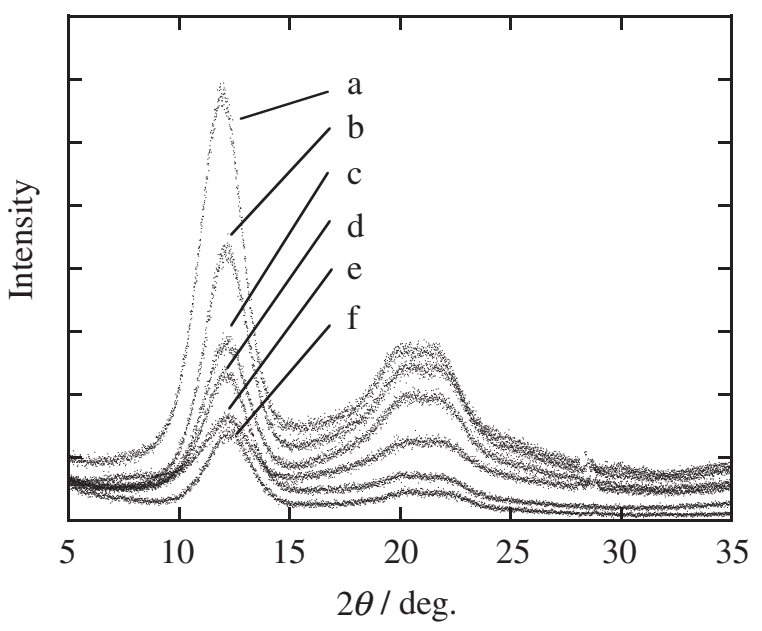

Figure 5. X-Ray diffraction patterns for cellophane treated with various hydrophobic solvents: a, cyclohexane; b, ethylacetate; c, toluene; d, carbon tetrachloride; e, DOP; f, aceton.

sometime during X-Ray diffraction measurement. The lowering of intensity is obviously due to the decrease in cellulose weight fraction. Therefore, analysis on the diffraction intensities might give information on the degree of interaction between cellulose and solvent. The order of intensity was cyclohexane $>$ ethylacetate $>$ toluene $>$ carbon tetrachloride $>$ DOP $>$ acetone. Acetone, which might have both hydrophobic and hydrophilic nature, interacts with cellulose most strongly, being resistant to vaporization from cellulose. Table II summerizes X-Ray parameters for cellophane treated with various solvents and physical parameters. All solvents had some potential to decrease $f_{\|(1 \overline{1} 0)}$ of cellophane, with slight lowering in diffraction angle for (110) plane. DOP and toluene strongly decreased the planar orientation index. Acetone, cyclohexan and carbon tetra-chloride has somewhat lesser potentials. However, their correlations with physical properties of solvents are not seen. Solvents having aromatic rings seem to give strong potentials for interacting to cellulose. Inspection of the ratio between RI for (110) and (020) gives a one-toone correlation, denoting that no specific influence on the planes takes place. Here, again the (110) plane seems to be widen by any of the solvents.

Table II. X-Ray parameters for cellophane treated with various solvents and physical parameters

\begin{tabular}{|c|c|c|c|c|c|c|c|c|c|c|c|c|}
\hline \multirow{2}{*}{ Solvent } & \multicolumn{4}{|c|}{ Solvent parameters } & \multicolumn{3}{|c|}{$(1 \overline{1} 0)$} & \multicolumn{2}{|c|}{$(110)$} & \multicolumn{2}{|c|}{$(020)$} & \multirow{2}{*}{$\begin{array}{c}f_{\|(1 \overline{1} 0)} \\
\text { after } 2 \mathrm{~h}\end{array}$} \\
\hline & $M_{\mathrm{W}}{ }^{\mathrm{a}}$ & $\varepsilon^{\mathrm{b}}$ & $\eta^{\mathrm{c}}$ & $\rho^{\mathrm{d}}$ & $2 \theta(\mathrm{deg})$ & $\operatorname{ACS}(\AA)$ & RI & $2 \theta(\operatorname{deg})$ & RI & $2 \theta(\mathrm{deg})$ & RI & \\
\hline Cyclohexane & 78 & 2.05 & 0.898 & 0.779 & 12.10 & 34.9 & 100 & 20.33 & 26.5 & 21.74 & 27.1 & 0.938 \\
\hline EtAc & 74 & 6.68 & 0.385 & 0.934 & 11.97 & 34.9 & 100 & 20.41 & 41.5 & 21.44 & 42.5 & 0.903 \\
\hline Toluene & 91 & 2.24 & 0.587 & 0.867 & 12.17 & 34.9 & 100 & 20.50 & 57.9 & 21.87 & 59.6 & 0.866 \\
\hline $\mathrm{CCl}_{4}$ & 154 & 2.24 & 0.965 & 1.595 & 11.75 & 34.9 & 100 & 20.08 & 18.4 & 21.51 & 20.4 & 0.955 \\
\hline DOP & 391 & 5.3 & 81.0 & 1.482 & 11.86 & 34.9 & 100 & 20.24 & 68.0 & 21.38 & 76.0 & 0.833 \\
\hline Acetone & 58 & 20.7 & 0.316 & 0.785 & 11.97 & 34.9 & 100 & 20.18 & 23.3 & 21.44 & 23.3 & 0.946 \\
\hline
\end{tabular}

${ }^{\mathrm{a}} M_{\mathrm{w}}$, molecular weight; ${ }^{\mathrm{b}} \varepsilon$, dielectric constant at $25^{\circ} \mathrm{C} ;{ }^{\mathrm{c}} \eta$, viscosity (poise); ${ }^{\mathrm{d}} \rho$, density $\left(\mathrm{g} / \mathrm{cm}^{3}\right)$. 


\section{CONCLUSIONS}

Analysis on X-Ray diffraction intensity of cellulose/solvent systems provided information on the degree of interaction between cellulose and solvent. Hydrophobic solvents are able to decrease (110) planar orientation index $\left(f_{\|(1 \overline{1} 0)}\right)$ for highly plane-orientated cellophane. Toluene, a hydrophobic solvent, caused concentration-specific decrease in $f_{\|(1 \overline{1} 0)}$ at toluene content of $c a$. 20-40 wt \% to cellulose with keeping its crystallinity and the degree of intermolecular hydrogen bonding. The molecular sheet-like assembly (corresponding to $\alpha_{\mathrm{sh}}$ relaxation), proposed previously by Yamane et al. ${ }^{9}$ may explain the present results. However, it is not yet clear whether such solvent concentration-specific change in orientation index is related to long-periodicity change, as observed for water. For this purpose, more detailed studies, such as small-angle X-Ray analysis and NMR relaxation analysis, should be made.

\section{REFERENCES}

1. C. Yamane and K. Okajima, Sen-i Gakkaishi, 53, 321 (1997).

2. H. Ono, M. Inamoto, K. Okajima, and Y. Yaginuma, Cellulose, 4, 57 (1997).

3. M. Ago, T. Endo, and T. Hirotsu, 10th annual meeting, Cellulose Society, Japan, Osaka, Preprint, p 63 (2003).
4. for example, H. Staudinger, K.-H. In der Birken, and M. Staudinger, Makromol. Chem., 9, 148 (1953).

5. G. N. Marchenko, V. N. Marsheva, V. I. Kovalenko, Ye. M. Belova, G. M. Khrapovskii, N. G. Gustova, and V. F. Sopin, Polym. Sci. USSR, 25, 1647 (1983).

6. C. Yamane and K. Okajima, Japan Patent 2001-331118 (2001).

7. T. Endo, M. Ago, Y. Shinohara, and T. Hirotsu, Polym. Prepr. Jpn., 51, (2002) IIIPe041.

8. M. Sasahira, C. Yamane, and K. Okajima, Cellulose Commun., 10, No. 1, 2 (2003).

9. C. Yamane, M. Mori, M. Saito, and K. Okajima, Polym. J., 28, 1039 (1996).

10. M. Ago, T. Endo, and T. Hirotsu, Unpublished results (Private correspondence; to be submitted to elsewhere).

11. K. Sato, H. Mochizuki, H. Ono, K. Okajima, and C. Yamane, to be submitted to Polym. $J$.

12. a) T. Takahashi, Sen-i Gakkaishi, 25, 80 (1969).

b) T. Takahashi, Sen-i Gakkaishi, 25, 122 (1969).

13. P. Scherrer, Göttinger Nachr., 98 (1918).

14. P. Langan, Y. Nishiyama, and H. Chanzy, Biomacromolecules, 2, 410 (2001).

15. Y. Ikada, Hyomen (Surface), 17, 769 (1979).

16. P. H. Hermans and A. Weidinger, J. Appl. Phys., 19, 491 (1948).

17. C. Yamane, T. Aoyagi, and K. Okajima, 10th annual meeting, Cellulose Society, Japan (Osaka), Preprint, p 77 (2003).

18. R. Fujioka and S. Manabe, '99 Pusan-Kyeongnam/KyushuSeibu Joint Symposium on High Polymers (9th) and Fibers (7th), Gyeongszng National University, Chinju, Korea, November 2, Proceedings, pp 102-105 (1999). 